\title{
Influenza outbreak - how best to prevent beyond vaccination?
}

\author{
Carlos Anjo, Gonçalo Coutinho, Pedro Vasconcelos, Glória Nunes da Silva, António Pais de Lacerda \\ Hospital Pulido Valente, Centro Hospitalar Lisboa Norte, Lisbon, Portugal
}

Received: January 15, 2019

Accepted: March 11, 2019

Online Published: March 15, 2019

DOI: $10.5430 /$ crim.v6n2p1

URL: https://doi.org/10.5430/crim.v6n2p1

\begin{abstract}
Background: Influenza vaccination, is the cornerstone of influenza epidemics prophylaxis, but there is some evidence of its lower efficacy in the elderly and in some other high-risk groups of the population.

Aims and Methods: We analysed the demographic characteristics of the patients (pts) hospitalized in an internal medicine ward (IMw) during the 2016-17 flu season, investigating whether there had been previous prevention, and overall how the infection progressed. All pts admitted to our hospital center, with positive nasopharyngeal swab, from the 1st October 2016 to the 28th February 2017 were recorded $(n=221)$, from which the inpatient IMw subgroup (31 pts) was selected.

Summary of results: Of the 31 selected IMw pts, $61.3 \%$ were female and $38.7 \%$ male. Their chronic main medical condition included diabetes mellitus type $2(32.3 \%)$ and heart failure (22.6\%). Although more than half was previously vaccinated with that year's vaccine (65.5\%), all pts required hospitalization, with a high average length of stay (11.3 \pm 5.2 days). H3N2 was the predominant subtype (90.3\%). Flu symptoms at admission were present in $80.6 \%$ of the pts, and their mean $\mathrm{PaO}_{2} / \mathrm{FiO}_{2}$ ratio was $288.9 \pm 40.7$. We observed an increased in infection severity in patients with asthma, obesity and chronic kidney disease (when compared to controls) with $\mathrm{PaO}_{2} / \mathrm{FiO}_{2}$ ratio $284.0 \pm 58.7,95 \% \mathrm{CI} 229.9-338.6, \mathrm{PaO}_{2} / \mathrm{FiO}_{2}$ ratio $242.5 \pm 12.0 ; 95 \% \mathrm{CI}$ 134.5-350.5 and $\mathrm{PaO}_{2} / \mathrm{FiO}_{2}$ ratio $263.0 \pm 33.9 ; 95 \% \mathrm{CI}-41.9-567.9$ respectively. No transfers were needed to intensive care, and one only patient required non-invasive mechanical ventilation.

Conclusions: During a flu epidemic, there is a constant need for health staff to perform the influenza test in all seeking care pts with respiratory / generalized pains complaints. Preventive use of oseltamivir by the most vulnerable contacts exposed to the virus, even if previously vaccinated, appears to be justifiable.
\end{abstract}

Key Words: Influenza outbreak, H3N2, Vaccination

\section{INTRODUCTION}

Influenza is caused by a virus primarily affecting the upper respiratory tract and sometimes the lungs, touching about 5\%$15 \%$ of each community population, and leading to $250,000-$ 500,000 deaths worldwide each year, mostly in the elderly cluster. ${ }^{[1]}$ Influenza vaccination, annually modified according to the prediction of the infectious virus in each coming flu season, is the cornerstone of prophylaxis, but there is some evidence of its lower efficacy in the elderly ${ }^{[2,3]}$ and in some other high-risk groups with previous cardiovascular and respiratory comorbidities. ${ }^{[3]}$ The influenza season 2016/17 started early in the northern and western European regions, being the earliest start of an influenza season since 2009/10. In Europe, the season was predominantly caused by A (H3N2) viruses, a subtype associated with more severe outcomes. ${ }^{[4]}$ Prophylactic use of Oseltamivir of at-risk and

\footnotetext{
*Correspondence: Carlos Anjo; Email: carloslccanjo@gmail.com; Address: Medicina III Hospital Pulido Valente, Alameda das Linhas de Torres, 117, 1769-001, Lisbon, Portugal.
} 
elderly groups might be considered in individual cases when influenza vaccination has not been performed, when not appropriate or ineffective because of virus strain mismatch, when influenza is circulating in the community and when contact with an infected person could not be avoided by other measures. ${ }^{[5]}$ We aimed to characterize the internal medicine hospital burden and severity of pandemic influenza across different age groups. Our specific objectives were to determine the characteristics of the population infected in 2016/2017 season, considering also the comorbidities, virulence of the virus, reason for hospital admission and complications during hospitalization. We considered also the prophylactic use of Oseltamivir in specific population groups.

\section{MATERIALS AND METHODS}

We analyzed the demographic characteristics of the patients hospitalized in an internal medicine ward during the 2016-17 flu season, assessing whether there had been previous prevention, and overall how their infection progressed clinically during their hospitalization. All patients with a positive rapid influenza diagnostic test, admitted to our hospital from the 1st October 2016 to the 28th February 2017, were recorded $(n=221)$, of which a subgroup of patients was selected ( $n$ =31) from the internal medicine ward. A descriptive study was made, all patients data being retrospectively analysed. Results from continuous variables are presented as mean standard deviation (SD) and 95\% confidence interval, categorical variables data are presented as percentages. $\mathrm{PaO}_{2} / \mathrm{FiO}_{2}$ ratio were compared between comorbidities and controls. Statistics were performed using the SPSS (version 23.0, Chicago, IL) software.

\section{Results}

Of the 31 selected internal medicine ward patients, $61.3 \%$ were female and $38.7 \%$ male. Their main chronic known medical conditions were diabetes mellitus type 2 (32.3\%), bronchial asthma (22.6\%); chronic obstructive pulmonary disease (COPD) (22.6\%) and congestive heart failure (22.6\%). Although more than $50 \%$ were previously vaccinated with that year's vaccine $(65.5 \%)$, all patients required hospitalization, with a high average length of stay (11.3 \pm 5.2 days). H3N2 was the predominant strain subtype (90.3\%). Flu symptoms at admission were present in $80.6 \%$ of the patients, and their mean $\mathrm{PaO}_{2} / \mathrm{FiO}_{2}$ ratio was $288.9 \pm 40.7$, with only mild apparent pulmonary repercussion. None had to be transferred to an intensive care unit, and only one patient required non-invasive mechanical ventilation at the ward.

Thirty-one hospitalized patients were enrolled in the study (mean age $73.9 \pm 17.9$ years old). Figure 1 shows the dis- tribution by age groups: 9 patients $(29.0 \%)$ were in the age group of 75 to 84 years, the most prevalent one (see Figure 1). Overall, of the 31 patients, $25(80.6 \%)$ were older than 65 years (see Figure 2). Twenty patients $(65.5 \%)$ had been vaccinated with that season's flu vaccine. The mean hospital stay of the patients was $11.3 \pm 5.2$ days. At admission all patients were treated with oseltamivir (75 mg 12/12h) for 5 days. ${ }^{[6]}$

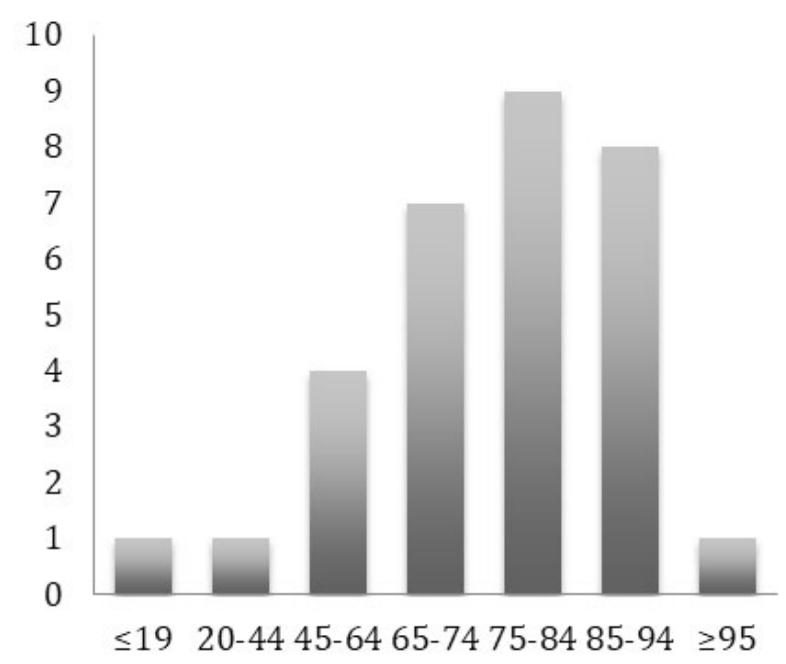

Figure 1. Patient's age groups

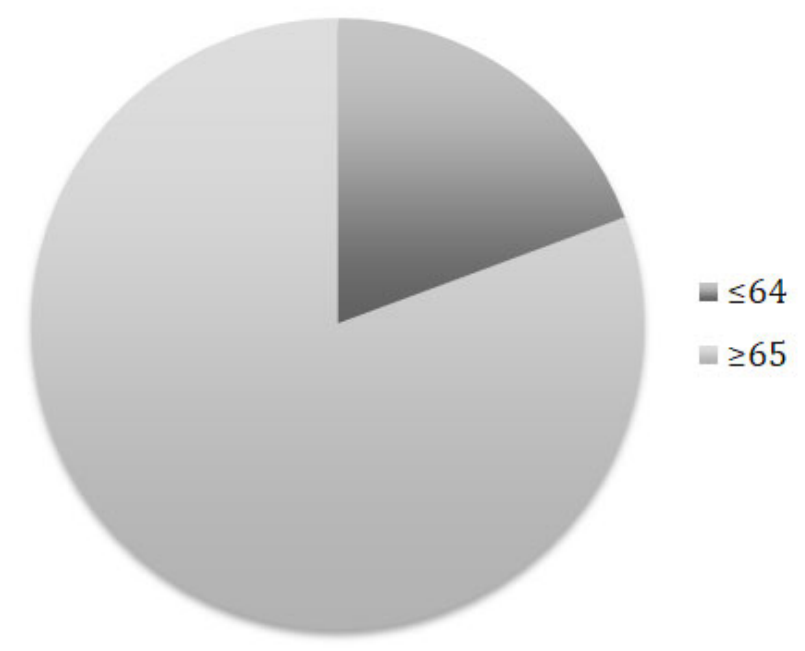

Figure 2. Patient's age by extended groups

In addition to respiratory symptoms caused by the influenza virus, patients had previous co-morbidities (see Table 1), some of which were more relevant to determine the severity and evolution of their disease. Hypertension was the most prevalent one $(64.5 \%)$. Other respiratory system comorbidities as chronic obstructive pulmonary disease (COPD) $22.6 \%$; asthma - 22.6\%; and previous bronchiectasis $(3.2 \%)$ have made viral infection more serious in these patients. 
Other chronic comorbidities that influenced the prognosis were: diabetes mellitus type $2-32.3 \%$, bronchial asthma - 22.6\%; heart failure - 22.6\%; neoplastic disease - $16.1 \%$; chronic kidney disease $-6.5 \%$; and coronary heart disease $32 \%$. Both obesity (IMC $>30 \mathrm{~kg} / \mathrm{m}^{2}$ ) and smoking habits were not very prevalent in our series - 6.5\% (see Table1). We observed an increased in infection severity in patients with asthma, obesity and chronic kidney disease (when compared to controls) with $\mathrm{PaO}_{2} / \mathrm{FiO}_{2}$ ratio $284.0 \pm 58.7,95 \%$ IC 229.9-338.6, $\mathrm{PaO}_{2} / \mathrm{FiO}_{2}$ ratio $242.5 \pm 12.0$; 95\% IC 134.5350.5 and $\mathrm{PaO}_{2} / \mathrm{FiO}_{2}$ ratio $263.0 \pm 33.9 ; 95 \% \mathrm{IC}-41.9-567.9$ respectively. The $\mathrm{PaO}_{2} / \mathrm{FiO}_{2}$ ratio differences are described in Table 2. The most prevalent influenza strain subtype was H3N2 - 28 patients $(90.3 \%)$. In tree patients $(9.7 \%)$ the subtype was undetermined. Twenty-five patients had on admission respiratory symptoms, general malaise and generalized pain, presenting a mean $\mathrm{PaO}_{2} / \mathrm{FiO}_{2}$ ratio of $288.9 \pm$ 40.7 (ambient air). Only one patient required non-invasive ventilation. All patients required hospitalization and none of them had to be admitted to intensive care. One patient died (mortality rate $-3.2 \%$ ).

Table 1. Patient's Comorbidities. Values of categorical variables are expressed as number and percentage

\begin{tabular}{ll}
\hline Comorbidities & N =90 \\
\hline Hypertension & $20(64.5)$ \\
Dyslipidemia & $10(32.3)$ \\
Type 2 Diabetes Mellitus & $10(32.3)$ \\
Asthma & $7(22.6)$ \\
Chronic obstructive pulmonary disease & $7(22.6)$ \\
Heart Failure & $7(22.6)$ \\
Neoplasm & $5(16.1)$ \\
Epilepsy & $2(6.5)$ \\
Hypothyroidism & $4(12.9)$ \\
Atrial fibrillation & $4(12.9)$ \\
Cerebrovascular disease & $2(6.5)$ \\
Parkinson's Disease & $2(6.5)$ \\
Obesity & $2(6.5)$ \\
Chronic kidney disease & $2(6.5)$ \\
Smoker & $2(6.5)$ \\
Bronchiectasis & $1(3.2)$ \\
Coronary heart disease & $1(3.2)$ \\
Sarcoidosis & $1(3.2)$ \\
Pacemaker & $1(3.2)$ \\
\hline
\end{tabular}

Most patients were admitted for respiratory infections (26 patients; $83.9 \%$ ). Community-acquired pneumonia was responsible for the admission of 2 patients, and 1 patient was admitted with global respiratory insufficiency.

Eighteen patients $(58.1 \%)$ had at least one complication. In fact in 16 patients $(88.9 \%)$ there was overlap of bacterial infection, one patient developed acute on chronic kidney injury and yet another developed atrial fibrillation with high ventricular rate.

\section{Discussion}

In recent Influenza epidemics, $71 \%$ to $85 \%$ of seasonal flurelated deaths occurred in people over 65 years. Hospitalizations due to these epidemics occurred in a high percentage $(54 \%-70 \%)$ in this same group. ${ }^{[7]}$ In our study it was also in the group of more than 65 years old that a greater number of patients with a need for hospital admission was verified (25 patients $-80.6 \%$ of the study population). Our study also confirms an increased in infection severity in patients with asthma, obesity and chronic kidney disease (when compared to controls) (see Table 2), this data is confirmed by some 2009/10 flu pandemic studies. ${ }^{[8]}$

Influenza A (H3N2) viruses are known to affect disproportionately those aged over 65 years, leading to outbreaks in long-term care homes, and a rise in hospitalization and mortality in this age group. ${ }^{[8]}$ Influenza vaccine effectiveness, among patients aged 65 years and older, has been low at both primary care $(23.4 \%)$ and hospital level $(2.5 \%){ }^{[3]}$

Despite all patients received a standard dose trivalent inactive influenza vaccine, there are other vaccines available (adjuvanted, high-dose) that could increase effectiveness. Although the high vaccination coverage (20 patients, $65.5 \%$ ), all these patients had required hospitalization, with a considerable length of stay (11.3 \pm 5.2 days), probably based on the low Influenza vaccine effectiveness, the risk of antiviral resistance with the widespread use of oseltamivir and the high number of comorbidities in this age group (see Table 1), leading to an increased risk of hospital complications. The use of oseltamivir as a prophylactic agent for periods of 5 days to 6 weeks confers a $58 \%-89 \%$ protection against influenza virus in healthy adults and $92 \%$ protection in a vaccinated elderly population. ${ }^{[5,9]}$

Therefore, the preventive use of oseltamivir in the most vulnerable contacts exposed to the virus, even if previously vaccinated, appears to be justifiable. One only patient died. He was an obese man with chronic obstructive pulmonary disease and heart failure who died in septic shock. In our group of patients hospitalized in an internal medicine ward for repercussions of influenza virus infection, there was a very low morbidity. ${ }^{[10]}$ In fact, there was no need for mechanical ventilation in an intensive care unit, ${ }^{[11]}$ and only one case required the institution of non-invasive mechanical ventilation. 
Table 2. $\mathrm{PaO}_{2} / \mathrm{FiO}_{2}$ ratio (comorbidities vs. controls). Continuous variables are expressed as average \pm standard deviation.

\begin{tabular}{lll}
\hline & \multicolumn{2}{c}{$\mathbf{P a O}_{2} / \mathbf{F i O}_{2}$ ratio } \\
\cline { 2 - 3 } & Comorbidities & Controls \\
\hline Type 2 Diabetes Mellitus & $299.4 \pm 34.7 ; 95 \%$ CI 274.6-324.2 & $283.9 \pm 43.1 ; 95 \%$ CI 264.3-303.6 \\
Asthma & $284.2 \pm 58.7 ; 95 \%$ CI 229.9-338.6 & $290.3 \pm 35.3 ; 95 \%$ CI 275.4-305.2 \\
Chronic obstructive pulmonary & $292.1 \pm 41.6 ; 95 \%$ CI 253.7-330.6 & $287.6 \pm 41.3 ; 95 \%$ CI 270,6-305,4 \\
disease & $300.4 \pm 41.0 ; 95 \%$ CI 262.5-338.4 & $285.6 \pm 40.8 ; 95 \%$ CI 268,3-302,8 \\
Heart Failure & $320.0 \pm 31.1 ; 95 \%$ CI 281.4-358.6 & $283.0 \pm 40.0 ; 95 \%$ CI 266.8-299.1 \\
Neoplasm & $263.0 \pm 33.9 ; 95 \%$ CI -41.9-567.9 & $290.7 \pm 41.0 ; 95 \%$ CI 275.1-306.3 \\
Chronic kidney disease & $318.0 \pm 53.7 ; 95 \%$ CI -164.8-800.8 & $286.9 \pm 40.1 ; 95 \%$ CI 271.7-302.2 \\
Smoker & $242.5 \pm 12.0 ; 95 \%$ CI 134.5-350.5 & $292.1 \pm 40.0 ; 95 \%$ CI 276.9-307.4 \\
Obesity &
\end{tabular}

\section{Conclusion}

In our study, we managed to characterize a population infected with the influenza virus, admitted to our hospital during the 2016-2017 season. Age group of people over 65 was the most affected. Despite the high prevalence of vaccination, a low Influenza vaccine effectiveness was observed. The virulence of this virus was low in this flu season. The most frequent complication was bacterial infection, for which the long periods of hospitalization certainly contributed. Despite a low morbidity and mortality, we've observed an increased in infection severity in patients with asthma, obesity and chronic kidney disease (when compared to controls). During a flu epidemic, there is a constant need for health staff to perform the influenza test in all seeking care patients with respiratory or generalized pains complaints, particularly if they are elderly or has multiple co-morbidities. The preventive use of oseltamivir in the most vulnerable close contacts exposed to the virus, even if they are already vaccinated, appears to be justifiable.

\section{CONFlicts OF InTEREST Disclosure}

The authors have declared no conflicts of interest.

\section{REFERENCES}

[1] Hayward AC. Comparative community burden and severity of seasonal and pandemic influenza: results of the Flu Watch cohort stud. Lancet Respir Med. 2014; 2: 445-454. https ://doi .org/10.101 6/S2213-2600 (14)70034-7

[2] Puig-Barberà J, García-de-Lomas J, Díez-Domingo J, et al. Influenza Vaccine Effectiveness in Preventing Influenza A(H3N2)-Related Hospitalizations in Adults Targeted for Vaccination by Type of Vaccine: A Hospital-Based Test-Negative Study, 2011-2012 A(H3N2) Predominant Influenza Season, Valencia, Spain. PLoS ONE. 2014; 9(11): e112294. https://doi.org/10.1371/journal. pone.0 112294

[3] Kissling E, Rondy M. Early 2016/17 vaccine effectiveness estimates against influenza $\mathrm{A}(\mathrm{H} 3 \mathrm{~N} 2)$ : I-MOVE multicentre case control studies at primary care and hospital levels in Europe. Euro Surveill. 2017; 22(7). https://doi.org/10.2807/1560-7917.ES.2017.22.7 .30464

[4] Melidou A, Broberg E. Predominance of influenza A(H3N2) virus genetic subclade 3C.2a1 during an early 2016/17 influenza season in Europe - Contribution of surveillance data from World Health Organization (WHO) European Region to the WHO vaccine composition consultation for northern hemisphere 2017/18. Vaccine. 2017; 35: 48284835. https://doi.org/10.1016/j . vaccine.2017.07.057

[5] Michiels B, Van Puyenbroeck K, Verhoeven V, et al. The Value of Neuraminidase Inhibitors for the Prevention and Treatment of Seasonal Influenza: A Systematic Review of Systematic Reviews. PLoS ONE. 2013; 8(4): e60348. https://doi.org/10.1371/journa 1. pone. 0060348
[6] Fiore AE. Antiviral agents for the treatment and chemoprophylaxis of influenza - recommendations of the Advisory Committee on Immunization Practices (ACIP). MMWR Recomm Rep. 2011; 60(1): 1-24. PMid:21248682.

[7] Kostova D, Reed C, Finelli L, et al. Influenza Illness and Hospitalizations Averted by Influenza Vaccination in the United States, 2005-2011. PLoS ONE. 2013; 8(6): e66312. https://doi.org/ 10.1371/journal.pone.0066312

[8] Adlhoch C, René S, Angeliki M, et al. The European Influenza Surveillance Network (2018). Dominant influenza A(H3N2) and B/Yamagata virus circulation in EU/EEA, 2016/17 and 2017/18 seasons, respectively. Euro Surveill. 2018; 23(13). https ://doi .org/ 10.2807/1560-7917.ES.2018.23.13.18-00146

[9] Mørland B, Fuglesang JE, Haaheim LR, et al. Effect of Oseltamivir (Tamiflu ${ }^{\circledR}$ ) for the Prevention and Treatment of Influenza During an Influenza Pandemic. NIPH Systematic Reviews: Executive Summaries. 2005.

[10] Biggerstaff M. Systematic Assessment of Multiple Routine and Near Real-Time Indicators to Classify the Severity of Influenza Seasons and Pandemics in the United States, 2003-2004 Through 20152016. American Journal of Epidemiology. 2018; 187(5): 1040-1050. https://doi.org/10.1093/aje/kwx334

[11] Reed C. Complications Among Adults Hospitalized With Influenza: A Comparison of Seasonal Influenza and the 2009 H1N1 Pandemic. Clinical Infectious Diseases. 2014; 59(2): 166-174. PMid:24785230. https://doi.org/10.1093/cid/ciu285 